\title{
Increased Collagen-linked Pentosidine Levels and Advanced Glycosylation End Products in Early Diabetic Nephropathy
}

\author{
Paul J. Beisswenger, Lynn L. Moore, Truls Brinck-Johnsen, and Thomas J. Curphey \\ Departments of Medicine and Pathology, Dartmouth Medical School, Hanover, New Hampshire 03755; \\ and the Dartmouth-Hitchcock Medical Center, Lebanon, New Hampshire 03756
}

\section{Abstract}

Rationale: Advanced glycosylation end products (AGEs) may play an important role in the development of diabetic vascular sequelae. An AGE cross-link, pentosidine, is a sensitive and specific marker for tissue levels of AGEs.

Objectives: To evaluate the role of AGEs in the development of diabetic nephropathy and retinopathy, we studied pentosidine levels and the clinical characteristics of 48 subjects with insulin-dependent diabetes mellitus. Diabetic nephropathy was classified as normal, microalbuminuria, or gross proteinuria, and retinopathy was graded as none, background, or proliferative.

Newly observed findings: Significant elevation of pentosidine $(P=0.025)$ was found in subjects with microalbuminuria or gross proteinuria (73.03 $\pm 9.47 \mathrm{vs} 76.46 \pm 6.37 \mathrm{pmol} / \mathrm{mg}$ col) when compared with normal $(56.96 \pm 3.26 \mathrm{pmol} / \mathrm{mg}$ col $)$. Multivariate analysis to correct for age, duration of diabetes, and gender did not modify the results. Elevated pentosidine levels were also found in those with proliferative when compared with those with background retinopathy $(75.86 \pm 5.66$ vs 60.42 $\pm 5.98 \mathrm{pmol} / \mathrm{mg} \mathrm{col})(P<0.05)$.

Conclusions: Microalbuminuria is associated with elevated levels of pentosidine similar to those found in overt diabetic nephropathy suggesting that elevated AGE levels are already present during the earliest detectable phase of diabetic nephropathy. (J. Clin. Invest. 1993. 92:212-217.) Key words: Maillard reaction - diabetic retinopathy $\bullet$ diabetic microangiopathy • Amadori reaction • microalbuminuria

\section{Introduction}

Increased nonenzymatic glycation of vascular and other tissues and the accumulation of advanced glycosylation end products (AGEs) ${ }^{1}$ are felt to play an important role in the development

This work was previously presented at the 52nd Annual Meeting of the American Diabetes Association, San Antonio, TX, 22 June 1992, and published in abstract form (1992. Diabetes. 41 [Suppl. 1]:9a.).

Address correspondence and reprint requests to Paul J. Beisswenger, M.D., Associate Professor of Medicine, Section of Endocrinology, Diabetes, and Metabolism, Dartmouth-Hitchcock Medical Center, 1 Medical Center Drive, Lebanon, NH 03756. Lynn L. Moore's current address is Boston University School of Medicine, Room B 612, 88 East Newton Street, Boston, MA 02118.

Received for publication 28 August 1992 and in revised form 19 January 1993.

1. Abbreviations used in this paper: AGEs, advanced glycosylation end products; $\mathrm{COV}$, coefficient of variation; FU, fluorescent unit.

J. Clin. Invest.

(C) The American Society for Clinical Investigation, Inc.

$0021-9738 / 93 / 07 / 212 / 06 \$ 2.00$

Volume 92, July 1993, 212-217 of diabetic vascular sequelae (1). The presence of AGEs on vascular structures is believed to be of particular importance in the pathogenesis of diabetic microvascular and macrovascular sequelae. The vascular changes associated with AGE accumulation could play a major role in the development and progression of tissue damage such as end-stage renal disease, retinal damage, and a host of biochemical and biological abnormalities associated with the accelerated atherosclerotic damage found in association with long-standing diabetes.

Although there is a substantial body of basic experimental data that supports the concept that AGEs play an important role in the development of diabetic sequelae, a limited number of clinical studies have been performed to assess the relationship between tissue levels of AGEs and the development of diabetic complications $(2,3)$. The studies conducted to date have primarily focused on subjects with advanced diabetic sequelae, and detailed examination of renal status and other characteristics have not been done in these study populations.

Our information on the relationship between the formation of AGEs in tissues and diabetic vascular sequelae has also been impeded by the limited number of assays available for the measurement of AGEs. Since many AGEs exhibit natural fluorescence, they have traditionally been measured by fluorimetry at several distinct excitation and emission wavelengths, although several new assays have recently been developed. These assays include one capable of detecting pentosidine, a specific AGE derived cross-link (4), as well as a radioreceptor/ELISA assay that can detect a larger family of AGEs (3). These assays are capable of detecting elevated levels of AGEs in blood or tissues, but they have not yet been studied for possible relationships with diabetic vascular sequelae in well-characterized populations of subjects with diabetes.

The goal of the current study is to examine the independent association between tissue levels of pentosidine and the renal and retinal sequelae of diabetes by performing a cross-sectional study of a population with diabetes. To detect larger families of AGEs, we have also measured these products with more traditional methods that detect fluorescence at $440 \mathrm{~nm}$ after excitation at $370 \mathrm{~nm}(5)$. Tissue levels of AGEs were studied in subjects with type I diabetes, who were comprehensively evaluated for diabetic sequelae and other clinical characteristics.

\section{Methods}

Subjects. The subjects in this study were selected sequentially from among the patients regularly attending the Diabetes Clinic at the Dartmouth-Hitchcock Medical Center. To be eligible to participate, subjects must have been 20-55 yr old, have type I diabetes as defined by the National Diabetes Data Group (6), have normal renal function based on a serum creatinine within the normal range (53-141 $\mu \mathrm{M}$ or $0.6-1.5 \mathrm{mg} / \mathrm{dl}$ ), and be willing to undergo a punch biopsy of the skin. The study, which was approved by the human studies committee, was explained, and informed consent was obtained.

50 willing and eligible subjects were entered into the study. We were 
unable to obtain an adequate skin biopsy on two of these subjects, leaving a total of 48 subjects in the current analyses.

We also recruited 29 healthy nondiabetic control subjects between the ages of 20 and 55 who were willing to undergo a skin biopsy.

Determination of diabetic sequelae. We carried out a complete history and physical examination and evaluated each subject for specific diabetes-related sequelae. A single retinal specialist (G. F.) examined each subject by direct and indirect dilated ophthalmoscopy to determine the degree of retinopathy. The specialist classified each subject's degree of retinopathy as none, background, preproliferative, or proliferative (7). To determine renal function, we collected two 24-h urine specimens to measure total protein, albumin, and creatinine clearance. Albumin was determined by RIA using a double antibody method (Diagnostic Products Corp., Los Angeles, CA), and total protein and creatinine clearance were determined by the clinical lab at the Dartmouth-Hitchcock Medical Center. Based on 24-h urinary albumin excretion, subjects were classified as normal $(<22 \mathrm{mg} / 24 \mathrm{~h}$ or $15 \mu \mathrm{g} /$ $\min )$, microalbuminuric $(22-150 \mathrm{mg} / 24 \mathrm{~h})$, or as having gross proteinuria ( $>150 \mathrm{mg} / 24 \mathrm{~h})(8)$.

Determination of advanced glycosylation end products in skin collagen. To determine the level of advanced glycosylation end products in collagen, a modification of the method of Monnier was used (2). Skin samples were obtained from the upper right buttock by a 4-mm punch biopsy and within $30 \mathrm{~min}$, they were snap frozen in liquid nitrogen. The epidermis was removed by sharp dissection under the dissecting microscope and after mincing, 4-15 mg of the tissue was further disrupted with a tissue homogenizer for $60 \mathrm{~s}$ (Polytron; Brinkmann Instruments, Inc., Westbury, NY). The tissue was then delipidated with chloroform-methanol $(2: 1)$ for $12 \mathrm{~h}$, washed, and suspended in $0.02 \mathrm{M}$ Hepes buffer $(\mathrm{pH}=7.5)$ containing $0.1 \mathrm{M}$ calcium chloride. The pellet was then digested in the same buffer containing $280 \mathrm{U}$ of type VII collagenase (Sigma Immunochemicals, St. Louis, MO) for $48-72 \mathrm{~h}$ at $37^{\circ} \mathrm{C}$ with constant shaking. The pellet remaining after digestion contained $<3 \%$ of the total tissue collagen from subjects with short or long duration diabetes and in those with and without vascular sequelae. To assay for collagen-linked fluorescence, samples were read in a spectrofluorimeter (model LS-3B Fluorescence Spectrometer; Perkin-Elmer Corp., Norwalk, CT) at an excitation wavelength of $370 \mathrm{~nm}$ with the reading of emissions at $440 \mathrm{~nm}$ (5). All readings were corrected for an enzyme blank, and hydroxyproline content was determined by the method of Stegemann and Stalder and converted to collagen assuming a hydroxyproline content of $14 \%$ (9). Fluorescence data are expressed as arbitrary fluorescent units per milligram of collagen. The within assay coefficient of variation (COV) for collagenlinked fluorescence was $6.2 \%$, and the between assay COV was $12 \%$ for autopsy control skin samples from the same subject assayed with each run.

Determination of pentosidine levels in skin collagen. For the determination of pentosidine, aliquots of the collagenase digests of skin were hydrolyzed in $6 \mathrm{~N} \mathrm{HCl}$ for $24 \mathrm{~h}$ at $110^{\circ} \mathrm{C}$ in vacuo. Calcium chloride was removed from the samples by precipitation with oxalic acid, the calcium oxalate precipitate was washed twice with water, and the supernatant and washes were pooled and taken to dryness using a speedvac system (Savant Instruments, Inc., Farmingdale, NY). The samples were then made up in mobile phase for the determination of pentosidine.

Pentosidine was determined by HPLC on a chromatograph (model 100A; Beckman Instruments, Inc., Fullerton, CA) using a $25 \mathrm{~cm}$ $\times$ 4.6-mm column (Spherisorb 5 ODS 2; Phenomenex Inc., Torrance, CA) and a mobile phase consisting of $25 \mathrm{mM}$ acetic acid, $25 \mathrm{mM}$ sodium acetate, $50 \mathrm{mM}$ sodium heptanesulfonate, and $14 \%(\mathrm{vol} / \mathrm{vol})$ acetonitrile-water at a flow rate of $1 \mathrm{ml} / \mathrm{min}$. Pentosidine was detected with a model 1046A fluorescence detector (Hewlett-Packard Co., Palo Alto, CA) connected to a model 4270 integrator (Spectra-Physics, Eugene, $O R$ ) at an excitation wavelength of $330 \mathrm{~nm}$, with the monitoring of emission at $390 \mathrm{~nm}$.

Quantitation. The amount of pentosidine present was determined by the external standards method using peak areas. Pentosidine stan- dards were kindly provided by Dr. Vincent Monnier of Case Western Reserve University (Cleveland, $\mathrm{OH}$ ). Standard solutions were prepared by appropriate dilution of a $10-\mu \mathrm{M}$ pentosidine standard into mobile phase buffer. Each standard was injected, as per the samples, at least three times during the course of a day's run. The areas for the standards were averaged, a standard curve was constructed, and peak areas were converted to concentration using a computer program to perform quadratic interpolation. The COV obtained for the standards (mean of 10 determinations) were $8 \%$ for the $20 \mathrm{nM}$ standard, $4.5 \%$ for the $100 \mathrm{nM}$ standard, and $2.2 \%$ for the $300 \mathrm{nM}$ standard.

Statistical methods. The goal of this analysis was to examine the relationship between tissue levels of AGEs and diabetic renal and retinal sequelae. We examined the tissue levels of AGEs by fluorescence at $440 \mathrm{~nm}$ after excitation at $370 \mathrm{~nm}$ and, more specifically, by determining the amount of pentosidine in the tissue. We classified the renal status of each subject into one of the three categories (normoalbuminuric, microalbuminuric, or gross proteinuric), and then examined the mean levels of tissue fluorescent products and pentosidine for the subjects in each category. The same univariate analyses were conducted examining mean tissue levels of AGEs for subjects with the three categories of retinopathy defined previously (none, background, and proliferative).

We used a standard one-way ANOVA to compare mean levels across groups. The $F$ test from the ANOVA procedure, the ratio of between-group variability to within-group variability, was used to test the null hypothesis of no difference in mean AGE or pentosidine levels among the groups (of diabetic complications). The $P$ value associated with the overall $F$ tests will be presented in the tables. When a statistically significant difference was found among the groups, a multiple comparisons procedure, Tukey's test, was used to make two-way comparisons between groups. However, the small numbers of subjects in some groups, and subsequent low power levels, could preclude firm conclusions from these post hoc comparisons for some of the subgroups. To adjust for the possible confounding effects of age, gender, and duration of diabetes, we employed an analysis of covariance to determine adjusted mean AGE levels for each category of nephropathy or retinopathy (10). Again, we present the $P$ values from the overall $F$ test from the analysis of covariance.

\section{Results}

Characteristics of the total sample. The mean age of the study population with diabetes was $36.1 \mathrm{yr}$ ( range $=21-51 \mathrm{yr}$ ) and that for control subjects was $36.1 \mathrm{yr}$ ( range $=20-52 \mathrm{yr}$ ). The mean duration of diabetes was $19.4 \mathrm{yr}$ ( range $=4-34 \mathrm{yr}$ ) as can be seen in Table I. $64 \%$ of the subjects were male, and $38 \%$ and $48 \%$ had retinopathy and nephropathy, respectively (Table I). As can be seen in Table II, the mean levels of albumin excretion for the subgroups with normoalbuminuria, microalbuminuria, and gross proteinuria were $6.33,42.17$, and 373.10

Table I. Characteristics of Total Sample

\begin{tabular}{lclc}
\hline Variable & $\begin{array}{c}\text { Mean } \pm \text { SD } \\
\text { (range) } \\
(n=48)\end{array}$ & $\begin{array}{c}\text { Gender } \\
\text { and sequelae }\end{array}$ & $\begin{array}{c}\text { Proportion } \\
\text { of total }\end{array}$ \\
\hline Age (yr) & $\begin{array}{c}36.1 \pm 8.4 \\
(21-51)\end{array}$ & Percent male & $64 \%$ \\
Controls (no diabetes) & $\begin{array}{c}36.1 \pm 8.8 \\
(20-52)\end{array}$ & $\begin{array}{c}\text { Percent with } \\
\text { retinopathy* }\end{array}$ & $38 \%$ \\
Duration diabetes (yr) & $\begin{array}{c}19.4 \pm 8.5 \\
(4-34)\end{array}$ & $\begin{array}{c}\text { Percent with } \\
\text { nephropathy }\end{array}$ & $48 \%$ \\
& & & \\
\end{tabular}

* Retinopathy, pre-proliferative or proliferative; ${ }^{\ddagger}$ Nephropathy, microalbuminuria or gross proteinuria. 
Table II. Nephropathy Status

\begin{tabular}{lccc}
\hline \multicolumn{1}{c}{$\begin{array}{c}\text { Nephropathy } \\
\text { category }\end{array}$} & $\begin{array}{c}\text { Number of } \\
\text { subjects }\end{array}$ & $\begin{array}{c}\text { Albuminuria } \\
\text { Mean } \pm \text { SE }\end{array}$ & $\begin{array}{c}\text { Creatinine clearance } \\
\text { Mean } \pm \text { SE }\end{array}$ \\
\hline & & $m g / 24 h$ & $m l / s$ \\
Normoalbuminuria & 24 & $6.33 \pm 0.58$ & $1.97 \pm 0.077$ \\
Microalbuminuria & 12 & $42.17 \pm 8.23$ & $1.83 \pm 0.088$ \\
Gross proteinuria & 12 & $373.10 \pm 150.7$ & $1.55 \pm 0.079$ \\
\hline
\end{tabular}

$\mathrm{mg} / 24 \mathrm{hr}$, respectively. When expressed as micrograms per minute these values were 4.40, 28.0, and 259.1. As also shown in Table II, the mean values for creatinine clearance in these three groups were $1.97 \mathrm{ml} / \mathrm{s}(117.9 \mathrm{ml} / \mathrm{min}$ ) (normoalbuminuria), $1.83 \mathrm{ml} / \mathrm{s}$ ( $109.5 \mathrm{ml} / \mathrm{min}$ ) (microalbuminuria), and $1.55 \mathrm{ml} / \mathrm{s}$ ( $93.3 \mathrm{ml} / \mathrm{min}$ ) (gross proteinuria). No significant relationship was found between creatinine clearance and tissue levels of pentosidine when examined by linear regression analy$\operatorname{sis}(P=0.21)$.

Pentosidine and AGE levels in diabetic and control subjects. When tissue levels of pentosidine (mean $\pm \mathrm{SE}$ ) were compared between subjects with and without diabetes (Fig. 1), we found a statistically significant $(P=0.0001)$ increase in pentosidine among subjects with diabetes $($ diabetes $=66.3 \pm 3.6$ vs controls $=31.2 \pm 2.2 \mathrm{pmol} / \mathrm{mg}$ collagen ). We also investigated the relation between age and the tissue levels of pentosidine using linear regression analysis separately for subjects with and without diabetes (Fig. 2). In the subjects with diabetes, we found that the tissue levels of pentosidine were higher for any given age (Fig. 2). In the control subjects, we found a significant positive relationship between age and tissue levels of pentosidine $(P$ $=.009$ ). Although the $P$ value associated with this relationship among subjects with diabetes was not as strong $(P=.082)$, the slope of the regression line (mean $\pm \mathrm{SE}$ ) was similar for the two groups $($ controls $=0.634 \pm 0.226$ and diabetes $=0.701 \pm 0.395$ $\mathrm{pmol} / \mathrm{yr}$ ). Finally, there was a statistically significant linear relationship between the duration of diabetes and pentosidine levels $(P=0.016)$ using a linear regression analysis; higher pentosidine levels were found in those with longer duration diabetes (data not shown). This relationship between the duration of diabetes and pentosidine levels was still of borderline significance $(P=0.054)$ when adjusted for the confounding effect of age by multiple regression analysis.

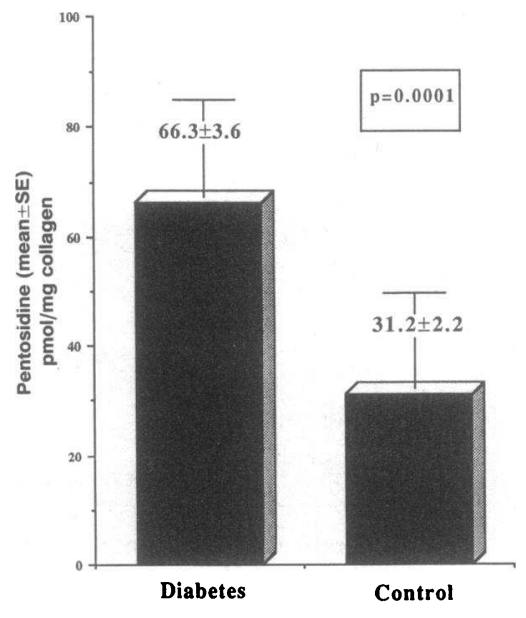

Figure 1. Pentosidine levels in subjects with and without diabetes. Significant differences were found for pentosidine levels in subjects with type 1 diabetes $(n$ $=48)$ and controls $(n$ $=29$ ).

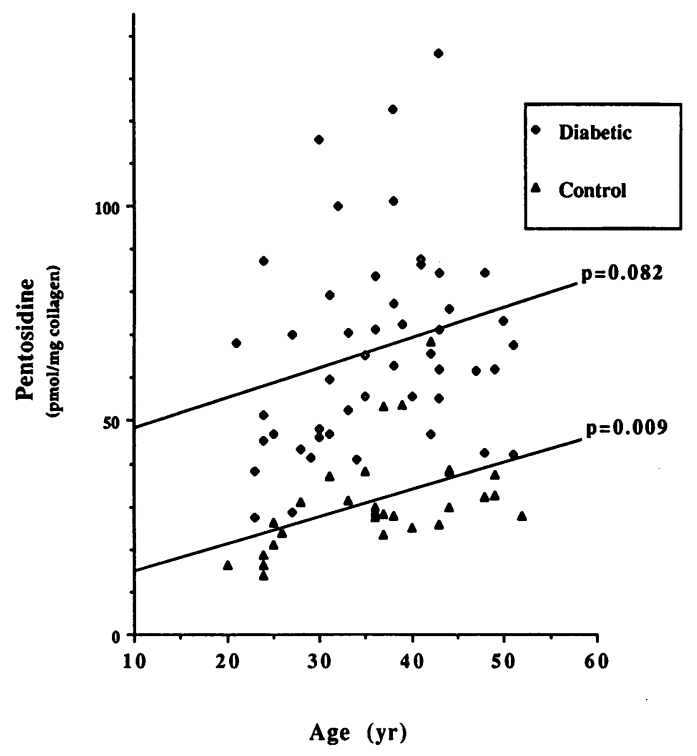

Figure 2. Changes in pentosidine with age. A significant increase in pentosidine with age is seen in subjects with type 1 diabetes (open diamonds) and nondiabetic controls (solid triangles). As can be seen by the slopes of the regression lines (controls $=0.634 \pm 0.226$ and diabetic $=0.701 \pm 0.395)$, the rate of increase with age is similar in both groups.

Pentosidine and AGE levels in subjects with diabetic nephropathy. When the relation between the degree of nephropathy and tissue levels of pentosidine was examined using ANOVA techniques ( Table III), we found a statistically significant difference in pentosidine levels (mean \pm SD) among subjects with normal albumin excretion $(56.6 \pm 16.3 \mathrm{pmol} / \mathrm{mg}$ collagen $)$, microalbuminuria (72.1 $\pm 30.1 \mathrm{pmol} / \mathrm{mg}$ collagen $)$, and gross proteinuria (79.2 $\pm 21.5 \mathrm{pmol} / \mathrm{mg}$ collagen). The $P$ value from the overall $F$ test examining differences across the three groups was 0.013 . It is interesting to note that pentosidine levels were very similar for subjects with microalbuminuria and

Table III. Mean Levels of Advanced Glycosylation End Products by Category of Diabetic Complication

\begin{tabular}{lcc}
\hline \multicolumn{1}{c}{ Diabtic complication } & $\begin{array}{c}\text { Pentosidine } \\
\text { Mean } \pm \text { SD }\end{array}$ & $\begin{array}{c}370 / 440 \mathrm{~nm} \\
\text { Mean } \pm \text { SD }\end{array}$ \\
\hline pmol/mg collagen & $F U / m g$ collagen \\
Renal status $(n=47)$ & & \\
$\quad$ Normoalbuminuria $(n=23)$ & $56.6 \pm 16.3$ & $8.9 \pm 2.9$ \\
Microalbuminuria $(n=12)$ & $72.1 \pm 30.1$ & $12.8 \pm 4.2$ \\
Gross proteinuria $(n=12)$ & $79.2 \pm 21.5$ & $13.1 \pm 3.5$ \\
$P$ value & 0.013 & 0.001 \\
Retinal Status $(n=48)$ & & \\
Normal $(n=13)$ & $59.8 \pm 15.6$ & $9.7 \pm 2.9$ \\
Background $(n=20)^{*}$ & $62.9 \pm 24.7$ & $10.0 \pm 3.6$ \\
Proliferative $(n=15)$ & $77.9 \pm 24.5$ & $12.9 \pm 4.6$ \\
$P$ value & 0.076 & 0.049 \\
& & \\
\hline
\end{tabular}

* Background retinopathy category includes three subjects with preproliferative retinopathy.

${ }^{\ddagger} P$ value from overall $F$ test from ANOVA. (Null hypothesis: no difference among group means). 
gross proteinuria. Based on the multiple comparisons procedure (Tukey's test), the pentosidine levels for both those with microalbuminuria and gross proteinuria differed significantly from those found in subjects with normoalbuminuria (Table III); the difference between the microalbuminuric and gross proteinuric groups was not, however, statistically significant. When tissue levels of AGEs measured by fluorescence at excitation/emission wavelengths of $370 / 440 \mathrm{~nm}$ were investigated using the $F$ test derived from ANOVA ( Table III), similar differences $(P$ value $=0.001)$ were seen overall between subjects with normal albumin excretion (8.9 \pm 2.9 fluorescent units (FU)/mg collagen ) and those with either microalbuminuria or gross proteinuria ( $12.8 \pm 4.2$ and $13.1 \pm 3.5 \mathrm{FU} / \mathrm{mg}$ collagen respectively). As was seen with pentosidine, the levels of AGEs measured by fluorescence were similar in subjects with microalbuminuria and gross proteinuria, while both groups differed significantly from those with normal albumin excretion when examined by the multiple comparisons procedure.

Pentosidine and AGE levels in subjects with diabetic retinopathy. The relationship between the retinopathy category and the tissue levels of pentosidine and fluorescent AGEs can be seen as well in Table III. Those with more advanced degrees of retinopathy (proliferative) had higher levels of pentosidine $(77.9 \pm 24.5 \mathrm{pmol} / \mathrm{mg}$ collagen) than those with either no detectable retinal change or background retinopathy (59.8 and $62.9 \mathrm{pmol} / \mathrm{mg}$ collagen, respectively), although these differences $(P=0.076)$ did not quite achieve significance at the $P$ $\leq 0.05$ level. Similar findings were seen when tissue levels of AGEs were measured as fluorescent products at excitation/ emission wavelengths of $370 / 440 \mathrm{~nm}$ (no retinopathy, 9.7; background retinopathy, 10.0; and proliferative retinopathy, 12.9 FU/mg collagen), although the difference across categories did achieve significance $(P=0.049)$. Pentosidine levels and fluorescent AGE levels were similar for subjects with no retinopathy and those with background retinopathy $(P>0.05)$ when examined by a multiple comparisons procedure ( $\mathrm{Tu}$ key's test).

Pentosidine and AGE levels adjusted for age, duration of diabetes, and gender. In Table IV, we present the results of the analysis of covariance used to adjust for potential confounding by age, gender, and duration of diabetes. As can be seen in this table, the adjusted mean levels of pentosidine and fluorescent AGEs (measured at excitation/emission wavelengths of 370/ $440 \mathrm{~nm}$ ) were similar in each category of nephropathy and retinopathy to the unadjusted levels, indicating little or no confounding by any of the three potential confounding factors. When adjusted pentosidine or fluorescent AGE levels were examined across the three categories of nephropathy, significant differences were found among those with normoalbuminuria, microalbuminuria, and gross proteinuria when examined by the $F$ test across groups $(P=0.010$ and 0.0008$)$ for pentosidine and fluorescent AGEs, respectively.

After adjusting for age, duration of diabetes, and gender, the relations between pentosidine and retinopathy $(P$ $=0.065)$, as well as fluorescent AGEs and retinopathy $(P$ $=0.041$ ), were similar to the earlier unadjusted results, although the $P$ value for the difference in pentosidine levels across retinopathy categories was again found to be of borderline significance. Using the adjusted data, those with either no retinopathy or with background retinopathy had lower pentosidine and fluorescent AGE values than those with proliferative retinopathy.
Table IV. Adjusted* Levels of Advanced Glycosylation End Products by Category of Diabetic Complication

\begin{tabular}{lcc}
\hline \multicolumn{1}{c}{ Diabetic sequelae } & $\begin{array}{c}\text { Pentosidine } \\
\text { Mean } \pm \text { SD }\end{array}$ & $\begin{array}{c}370 / 440 \mathrm{~nm} \\
\text { Mean } \pm \text { SD }\end{array}$ \\
\hline & pmol/mg collagen & $F U / m g$ collagen \\
Renal Function $(n=47)$ & & \\
$\quad$ Normoalbuminuria $(n=23)$ & $57.4 \pm 22.5$ & $9.1 \pm 3.6$ \\
Microalbuminuria $(n=12)$ & $68.6 \pm 21.5$ & $12.3 \pm 3.4$ \\
Gross proteinuria $(n=12)$ & $81.4 \pm 23.3$ & $13.1 \pm 3.7$ \\
$P$ value & 0.010 & 0.0008 \\
Retinal status $(n=48)^{\ddagger}$ & & \\
Normal $(n=13)$ & $68.1 \pm 30.4$ & $10.1 \pm 5.1$ \\
Background $(n=20)$ & $60.5 \pm 23.3$ & $10.1 \pm 3.9$ \\
Proliferative $(n=15)_{P \text { value }}^{\ddagger}$ & $73.8 \pm 24.5$ & $12.5 \pm 4.1$ \\
& 0.065 & 0.041 \\
\hline
\end{tabular}

* All mean levels were adjusted for age, gender, and duration of diabetes using an analysis of covariance procedure.

${ }^{\ddagger} P$ value from overall $F$ test from ANOVA. Null hypothesis: no difference among group means, after adjustment for age, gender, and diabetes duration.)

\section{Discussion}

Analysis of the levels of pentosidine and fluorescent advanced glycosylation end-products in our subjects with and without diabetes showed that diabetes is associated with a significant elevation of these products in skin collagen. A significant increase in pentosidine was also found with increasing age, as well as with increasing duration of diabetes. The relationship between pentosidine and age was highly significant for nondiabetic subjects but of borderline significance for those with diabetes. This may be secondary to the greater variability in pentosidine levels in those with diabetes which, in turn, could be related to the heterogeneity in the diabetic population regarding duration of diabetes and the level of vascular sequelae. Although the absolute levels of pentosidine were significantly increased in diabetes, the rate of increase in pentosidine with increasing age, as reflected in the slopes, was similar for those with and without diabetes (Fig. 2). This conflicts with the findings of an earlier study by Monnier et al. in which they found an accelerated rate of increase in tissue fluorescence with increasing age for those with diabetes relative to control subjects (11). The current study is, however, in agreement with a recently published study by Sell and Monnier, which also found that the rate of increase in pentosidine with age was similar for diabetic and control subjects (12). These studies suggest that increased tissue levels of pentosidine occurs fairly early in the diabetic syndrome, although studies of greater numbers diabetic subjects with short duration diabetes will be necessary to document the point at which this increase occurs.

The tissue levels of pentosidine and fluorescent AGEs were also found to be significantly elevated in subjects with diabetic nephropathy. However, when the relationship between retinopathy and AGEs was examined, the elevation of pentosidine and fluorescent AGE levels in those with proliferative retinopathy was marginally significant relative to those who had minimal or no evidence of significant retinal sequelae (Table III). The elevated levels of AGEs (and pentosidine) persisted after adjustment for differences in age, duration of diabetes, and 
gender by multivariate analysis (Table IV), suggesting that the relationships between the diabetes-related complications and tissue levels of AGEs or pentosidine are not significantly confounded by these factors. In addition to finding elevated levels of pentosidine and AGEs in the tissues of those with overt diabetic nephropathy, we also found that subjects with microalbuminuria had elevated pentosidine and fluorescent product levels relative to those with normal albumin excretion ( Tables III and IV). Conversely, levels of pentosidine and fluorescent AGEs in microalbuminuric subjects were similar to those seen in subjects with clinically more advanced degrees of diabetic nephropathy (gross proteinuria). Finding elevated levels of pentosidine and fluorescent AGEs in subjects with microalbuminuria, which is generally considered an earlier and potentially reversible phase of diabetic nephropathy $(8,13)$, suggests that generalized modification of tissue collagen may already be present during this phase of diabetic nephropathy. The finding of significant elevation of AGEs during the microalbuminuria phase of diabetic nephropathy also supports the findings of Chavers et al. which showed that significant changes in glomerular morphology were already present during this stage of nephropathy (14). In the current study, we purposely defined microalbuminuria using the most conservative criteria for abnormal albumin excretion to avoid selection of those with more advanced glomerulosclerosis (15). In spite of this selection process, elevated AGE levels were found in the group with microalbuminuria, as well as in those with more advanced degrees of proteinuria. It should also be noted, however, that collagen in vascular structures is presumably the site of pathology in diabetes, and that although skin collagen is similar to other collagens in its slow turnover (16), it is possible that tissue remodeling and receptor-mediated turnover processes may differ in different collagen containing tissues.

Our finding that significant elevation of pentosidine and fluorescent AGEs is associated with diabetic nephropathy does not agree with the data of Sell and Monnier. Although one study by the same authors showed significant elevation of tissue levels of pentosidine in association with end-stage renal disease in diabetic and nondiabetic subjects (17), other studies showed no significant relationship between diabetic nephropathy and tissue levels of fluorescent AGEs (2), or pentosidine in the absence of end-stage renal disease (12). In the later studies, the failure to demonstrate elevated AGEs in association with nephropathy may be partially caused by the method that they used to classify nephropathy. In these studies, they defined stage 0 nephropathy as urinary protein excretion of less than $500 \mathrm{mg} / 24 \mathrm{~h}$, which if classified by currently accepted methods, would include subjects with microalbuminuria and more advanced proteinuria $(8,15)$. In the current study, we have shown that tissue levels of pentosidine and fluorescent AGEs are significantly elevated in association with microalbuminuria and gross proteinuria (defined as 24-h urinary protein excretion of $22-150 \mathrm{mg} / 24 \mathrm{~h}$ or $>150 \mathrm{mg} / 24 \mathrm{~h}$, respectively). Because of the wide range of proteinuria in their 0 nephropathy group, it is quite likely that Sell et al. could have included a number subjects with significant albuminuria in this group, and this could spuriously increase AGE levels in the group that they defined as having minimal nephropathy. This could mask differences in tissue levels of AGEs between this group and those with greater degrees of proteinuria, and preclude finding a significant relationship between pentosidine or fluorescent AGEs and diabetic nephropathy.
When the relationship between diabetic retinopathy and the tissue levels of pentosidine and fluorescent AGEs was examined (Tables III and IV), we found that AGE levels in those with less advanced diabetic retinopathy (background) are similar to the levels seen in those with normal retinal exams, and that AGE levels in both of these subgroups are lower than those with more advanced grades of retinopathy (proliferative). Since the relationship between pentosidine and the degree of retinopathy was of borderline significance, and we were unable to demonstrate differences in the groups with no retinopathy and background retinopathy, our results suggest that elevated tissue levels of AGEs are less predictive of the earlier stages of retinopathy then for the earlier microalbuminuria stage of diabetic nephropathy. When the data were adjusted for age, duration of diabetes, and gender, the tissue levels of pentosidine were slightly lower in the group with background retinopathy than in those with no detectable retinopathy (Table IV), although these differences did not approach statistical significance. The moderately large variability around the mean pentosidine levels (as can be seen from the standard deviations presented in Table IV) and the relatively small sample sizes in some groups could account for this unexpected finding. Thus, it would be beneficial to examine larger numbers of subject with each type of complication in future studies (particularly with retinal complications) to derive more stable estimates of adjusted mean pentosidine levels. It is also possible that more sensitive methods for classifying diabetes-related retinal damage may be necessary to demonstrate significant differences in AGE levels between groups with earlier stages of retinopathy. Tissue levels of other specific AGEs or families of AGEs (1820) may also be more predictive of diabetes-associated retinopathic changes than pentosidine or AGEs measured by fluorescence.

In our study, as in previous studies (21), we found an increased risk of multiple diabetes-related complications in certain individuals; 12 of the 15 subjects with proliferative retinopathy also had evidence of diabetic nephropathy (eight with gross proteinuria and four with microalbuminuria). It is possible that this association of retinopathy with nephropathy and the significant relationship between nephropathy and tissue levels of AGEs could result in nephropathy having a confounding effect on the relationship between retinopathy and AGEs, although it is difficult to determine the importance of this association because of the small numbers of subjects with advanced retinopathy without concurrent nephropathy.

Although we found a definite association between AGE levels and the presence of diabetic renal sequelae and a possible association of AGEs and diabetic retinopathy in this cross-sectional study, this association does not prove that AGEs play a causal role in the development of vascular complications. There exists, however, a large body of data to support an important role for AGEs in the pathogenesis of diabetes-related vascular damage. Previous studies have demonstrated that the accumulation of these products on collagens and other proteins can produce structural and functional changes in vascular tissues by multiple mechanisms. Increased cross-linking of structural proteins such as collagens and basement membranes can lead to decreased compliance and rates of turnover of structural components of the vascular wall and can also lead to functional abnormalities, such as abnormal basement membrane filtration function (22). Abnormal autoaggregation of basement membrane collagenous subunits $(23,24)$, abnormal 
association of type IV collagen with other basement membrane components such as laminin, proteoglycans and intactin (25), as well as increased immunogenicity (26) and succeptibility to macrophage-induced damage (27) can all occur as a result of tissue modification by AGEs. AGE accumulation on, or in proximity to cellular components of the vascular wall, can also result in direct cytotoxicity or indirect macrophage/cytokine induced damage (27). AGE-associated modification of structural proteins in the capillary or arterial wall and dysfunction or death of cellular elements could lead to stiff, less compliant vessels with altered permeability and other functional characteristics and eventually, luminal occlusion from excessive accumulation of basement membrane material or basement membrane-protein adducts. These changes could play a major role in the development and progression of the tissue damage such as end-stage renal disease, retinal damage, and the host of biochemical and biological abnormalities associated with the accelerated atherosclerotic damage found in association with long-standing diabetes.

\section{Acknowledgments}

We would like to thank Linda Rockenmaker and Smith Jean for expert technical assistance and Gault Farrell M.D. (G. F.) for performing the retinal examinations.

The research was supported by grants from the Diabetes Research and Education Foundation, The Harriet Krusi Fund/Hitchcock Foundation Inc., and the Mildred Dole Trust.

\section{References}

1. Brownlee, M., A. Cerami, and H. Vlassara. 1988. Advanced Glycosylation end products in tissue and the biochemical basis of diabetic complications. $N$. Engl. J. Med. 318:1315-1321.

2. Monnier, V., V. Vishwanath, K. F. Frank, C. A. K. Elmets, P. Sauthot, and R. R. Kohn. 1986. Relation between complications of type I diabetes mellitus and collagen-linked fluorescence. N. Engl. J. Med. 314:403-408.

3. Makita, Z., S. Radoff, E. J. Rayfield, Z. Yang, E. Skolnik, V. Delany, E. A. Friedman, A. Cerami, and H. Vlassara. 1991. Advanced glycosylation end products in patients with diabetic nephropathy. N. Engl. J. Med. 325:836-841.

4. Sell, D. R., and V. M. Monnier. 1989. Structure elucidation of a senescence cross-link from human extracellular matrix. J. Biol. Chem. 263:3758-3764.

5. Sell, D. R., and V. M. Monnier. 1989. Isolation, purification and partial characterization of novel fluorophores from aging human insoluble collagen-rich tissue. Connect. Tissue Res. 19:77-92.

6. National Diabetes Data Group. 1979. Classification of diabetes mellitus and other categories of glucose intolerance. Diabetes. 28:1039.
7. ETDRS. 1980. ETDRS Coordinating center: manual of operations. Diabetes Retinopathy Coordinating Center, Baltimore, MD. Chapter II.

8. Viberti, G. 1988. Recent advances in understanding mechanisms and natural history of diabetic renal disease. Diabetes Care. 11:3-9.

9. Stegemann, H., and K. Stalder. 1967. Determination of hydroxyproline. Clin. Chim. Acta. 18:267-273.

10. Neter, J., W. Wasserman, and M. H. Kutner. 1985. Applied Linear Statistical Models. Richard D. Irwin, Inc., Homewood, IL. 661-672.

11. Monnier, V., C. A. Elmets, K. E. Frank, V. Vishwanath, and T. Yamashita. 1986. AGE-related normalization of the browning rate of collagen in diabetic subjects without retinopathy. J. Clin. Invest. 78:832-835.

12. Sell, D. R., A. Lapolla, O. Odetti, J. Fogarty, and V. M. Monnier. 1992. Pentosidine formation in skin correlates with severity of complications in individuals with long-standing IDDM. Diabetes. 41:1286-1292.

13. Mogensen, C. E. 1989. Natural history of renal functional abnormalities in human diabetes mellitus: from normalbuminuria to incipient and overt ne phropathy. In The Kidney in Diabetes Mellitus. B. M. Brenner and J. H. Stein, editors. Churchill Livingstone, Inc., New York. pp. 19-49.

14. Chavers, B. M., R. W. Bilous, M. R. C. P., E. N. Ellis, M. W. Steffes, and M. Mauer. 1989. Glomerular lesions and urinary albumin excretion in type I diabetes without overt proteinuria. $N$. Engl. J. Med. 320:966-70.

15. Mogensen, C. E. 1990. Prediction of clinical diabetic nephropathy in IDDM patients: alternatives to microalbuminuria? Diabetes. 39:761-767.

16. Molnar, J. A., N. M. Alpert, D. A. Wagner, S. Miyatani, J. F. Burke, and V. R. Young. 1988. Synthesis and degradation of collagens in skin of healthy and malnourished rats in vivo, studied by ${ }^{18} \mathrm{O}_{2}$ labelling. Biochem. J. 250:71-76.

17. Sell, D., and V. M. Monnier. 1990. End-stage renal disease and diabetes catalyze the formation of pentose-derived crosslink from aging human collagen. J. Clin. Invest. 85:380-384.

18. Makita, Z., H. Vlassara, A. Cerami, and R. Bucala. 1992. Immunochemical detection of advanced glycosylation end products in vivo. J. Biol. Chem. 267:5133-5138.

19. Radoff, S., Z. Makita, and H. Vlassara. 1991. Radioreceptor assay for advanced glycosylation end products. Diabetes. 40:1731-1738.

20. Miyata, S., and V. Monnier. 1992. Immunohistochemical detection of advanced glycosylation end products in diabetic tissues using monoclonal antibody to pyrraline. J. Clin. Invest. 89:1102-1112.

21. Krolewski, A. J., J. H. Warram, R. Kahn, L. I. Kahn, and C. R. Kahn. 1987. Epidemiologic approach to the etiology of type I diabetes mellitus and its complications. N. Engl. J. Med. 18:267-273.

22. Brownlee, M., S. Ponger, and A. Cerami. 1983. Covalent attachment of soluble proteins by nonenzymatically glycosylated collagen. J. Exp. Med. 158:1739-1744.

23. Yurchenco, P. D., and G. C. Ruben. 1987. Basement membrane structure in situ: evidence for lateral associations in the type IV collagen network. J. Cell Biol. 105:2559-2568.

24. Tsilibary, E., A. Charonis, L. Reger, R. Wohlhueter, and L. T. Furcht. 1988. The effect of non-enzymatic glycosylation on the main noncollagenous $\mathrm{NCl}$ domain to type IV collagen. J. Biol. Chem. 263:4302-4308.

25. Brown, D. M., C. A., L. Furcht, D. Klein, M. Mauer, M. Steffes, and P. Tsilibary. 1991. Review of matrix components. Diabetes Care. 14:157-159.

26. Bassiouny, A. R., H. Rosenberg, and T. L. McDonald. 1983. Glycosylated Collagen is Antigenic. Diabetes. 32:1182-1184.

27. Vlassara, H., M. Brownlee, and A. Cerami. 1985. High affinity receptor mediated uptake and degradation of glucose modified proteins: a potential mechanism for the removal of senescent macromolecules. Proc. Natl. Acad. Sci. USA. 82:5588-5592. 